\title{
Paraoxonase 1 Q192R (PON1-192) polymorphism is associated with reduced lipid peroxidation in healthy young men on a low-carotenoid diet supplemented with tomato juice
}

\author{
Achim Bub $^{1 *}$, Stephan W. Barth ${ }^{1}$, Bernhard Watzl ${ }^{1}$, Karlis Briviba ${ }^{1}$ and Gerhard Rechkemmer ${ }^{2}$ \\ ${ }^{1}$ Institute of Nutritional Physiology, Federal Research Centre for Nutrition and Food, Haid-und-Neu-Str. 9, Karlsruhe, Germany \\ ${ }^{2}$ Department of Food and Nutrition, Center of Life Science, Technical University of Munich, Freising-Weihenstephan, Germany
}

(Received 6 February 2004 - Revised 10 September 2004 - Accepted 21 September 2004)

The HDL-bound enzyme paraoxonase (PON) protects LDL from oxidation and may therefore attenuate the development of atherosclerosis. We examined the effect of tomato and carrot juice consumption on PON1 activity and lipid peroxidation in healthy young volunteers with different PON1-192 genotypes (Q/R substitution at position 192). In this randomized cross-over study twenty-two healthy, non-smoking men on a low-carotenoid diet received $330 \mathrm{ml} / \mathrm{d}$ tomato juice (37.0 mg lycopene, $1.6 \mathrm{mg} \beta$-carotene) or carrot juice $(27.1 \mathrm{mg} \beta$-carotene, $13.1 \mathrm{mg} \alpha$-carotene) for 2 weeks. Intervention periods were preceded by 2-week low-carotenoid intake. We determined the PON1-192 genotype by restriction fragment length polymorphism-polymerase chain reaction (RFLP-PCR) and measured ex vivo LDL oxidation (lag time), plasma malondialdehyde and PON1 activity at the beginning and end of each intervention period. At baseline, lag time was higher $(P<0 \cdot 05)$ in QQ (111 (SD 9) $\mathrm{min})$ than in QR/RR subjects (101 (SD 8) min). Neither tomato nor carrot juice consumption had significant effects on PON1 activity. However, tomato juice consumption reduced $(P<0.05)$ plasma malondialdehyde in QR/RR $(\Delta:-0.073$ (SD 0.11) $\mu \mathrm{mol} / \mathrm{l})$ as compared to QQ subjects $(\Delta:+0.047$ (SD 0.13) $\mu \mathrm{mol} / \mathrm{l})$. Carrot juice had no significant effect on malondialdehyde irrespective of the PON1-192 genotype. Male volunteers with the QR/RR genotype showed an increased lipid peroxidation at baseline. Although tomato and carrot juice fail to affect PON1 activity, tomato juice intake reduced lipid peroxidation in healthy volunteers carrying the R-allele of the PON1-192 genotype and could thus contribute to CVD risk reduction in these individuals.

Tomato juice: Antioxidants: Lipid peroxidation: Paraoxonase polymorphism

Diet plays an important role among the risk factors for atherosclerosis and CVD since it has an impact on, for example, plasma lipids, blood pressure and antioxidant mechanisms. Large prospective cohort studies revealed that a high intake of fruit and vegetables (Liu et al. 2000, 2001; Joshipura et al. 2001; Bazzano et al. 2002) and of dietary carotenoids from fruit and vegetables (Osganian et al. 2003) reduce CVD risk. In contrast, supplemental carotenoids from a non-food source have no beneficial effects on CVD risk and supplementation should not be further recommended (Vivekananthan et al. 2003).

Oxidation of LDL and the subsequent generation of lipid hydroperoxides and other lipid oxidation products are hypothesized to play a crucial role in the development of atherosclerosis and CVD (Diaz et al. 1997). Dietary antioxidants such as ascorbic acid, vitamin $\mathrm{E}$ and $\beta$-carotene can inhibit LDL oxidation in vitro (Frei et al. 1996; Jialal \& Devaraj, 1996) and may therefore protect from CVD although clinical trials testing antioxidant supplementation revealed conflicting results (Salonen, 2002; Upston et al. 2003). LDL oxidation is also reduced by paraoxonase (PON), an enzyme bound to HDL (Mackness et al. 1991, 2000a; Aviram et al. 1998), and may therefore attenuate the development of atherosclerosis (Mackness et al. 1998a; Aviram, 1999). Recent studies proposed that paraoxonase activity predicts CVD risk (Jarvik et al. 2000, 2003; Mackness et al. 2003). So far, it is not yet clear whether the activity polymorphism for PON1-192, with a Q/R substitution at position 192 (Adkins et al. 1993; Humbert et al. 1993), also has an impact on atherosclerosis (Mackness et al. 2003). The PON1 QQ-genotype exhibits low activity towards hydrolysis of paraoxon, while the RR-genotype shows high activity towards paraoxon. However, this isoenzyme corresponding to the RRgenotype exhibits a low hydrolysing activity towards lipid hydroperoxides. The phenylacetate substrate activity is not affected by the PON1-192 genotype (Mackness et al. 1998a).

PON1 activity can be modulated by dietary factors such as alcohol (vander Gaag et al. 1999), pomegranate juice (Aviram et al. 2000) and dietary fat (Sutherland et al. 1999; Kudchodkar et al. 2000; Mackness et al. 2000b; Tomás et al. 2001) and may thus have an impact on CVD risk. A high vegetable intake also lowers PON1 activity and this effect was most prominent in women with the PON1-192 QR/RR-genotype (Rantala et al. 2002). In a similar study, Kleemola et al. (2002) showed that PON1 activity is reduced during high vegetable intake and that it is negatively correlated with the intake of $\beta$-carotene. Data on other carotenoids, e.g. lycopene, were not presented in that study. 
Recently, we demonstrated that consumption of a lycopene-rich tomato juice for 2 months reduced lipid peroxidation in QR/RR elderly subjects, but had no effect on PON1 activity (Bub et al. 2002). Here we investigated whether tomato or carrot juice consumption modulates PON1 activity and parameters of lipid peroxidation in healthy young volunteers on a low-carotenoid diet. Furthermore, we determined the PON1-192 genotype in order to see whether possible intervention effects are related to the PON1-192 polymorphism.

\section{Material and methods}

\section{Subjects}

Twenty-two healthy, non-smoking men participated in this study. All subjects were in good health as was determined by a screening history and medical examination. Subjects on medication or taking supplements were excluded from the study. The study was approved by the Medical Ethics Committee of the Landesärztekammer Baden-Württemberg and all participants gave their consent in writing.

\section{Study design}

This study was designed as a randomized cross-over study of two intervention periods ( 2 weeks each) with tomato juice or carrot juice ( $330 \mathrm{ml} / \mathrm{d}$; Schoenenberger, Magstadt, Germany). Study participants were allocated to two groups (A: tomato juice/carrot juice; $\mathrm{B}$ : carrot juice/tomato juice). The tomato juice supplement $(330 \mathrm{ml} / \mathrm{d})$ provided $37.0 \mathrm{mg}$ lycopene and $1.6 \mathrm{mg} \beta$-carotene. Carrot juice supplementation $(330 \mathrm{ml} / \mathrm{d})$ supplied $27 \cdot 1 \mathrm{mg} \beta$-carotene and $13.1 \mathrm{mg} \alpha$-carotene. Intervention periods were preceded by 2-week low-carotenoid periods and a 2 -week washout period between the two interventions. After the second intervention period a third 2-week low-carotenoid period followed resulting in a total study period of 10 weeks. From a previous study we know that a 2-week depletion or supplementation period is long enough to induce significant changes in plasma carotenoid concentrations and lipid peroxidation (Bub et al. 2000). Subjects were told to consume the juices with their main meals and were not restricted in their daily diet, except that they had to abstain from fruit and vegetables high in carotenoids throughout the whole study period. A list of the fruit and vegetables the subjects were not allowed to eat was provided (Müller et al. 1999).

\section{Collection and preparation of blood samples}

Fasting blood samples were taken at the beginning of the study and at the end of each experimental week in the morning between 7.00 and 9.00 hours. Blood was drawn from an antecubital vein into prechilled tubes containing EDTA (1.6 g/l; Monovette-Sarstedt, Nümbrecht, Germany) and immediately placed on ice in the dark. Plasma was collected after centrifugation at $1500 \mathrm{~g}$ for $10 \mathrm{~min}$ at $4^{\circ} \mathrm{C}$. For the lipid peroxidation assay sucrose $(15 \mathrm{~g} / \mathrm{l})$ was added to the plasma to prevent LDL aggregation and stored at $-80^{\circ} \mathrm{C}$ until analysis. Tubes without anticoagulant (SerumMonovette-Sarstedt) were used for serum collections. Blood was allowed to clot at room temperature for $30 \mathrm{~min}$, then centrifuged at $1500 \mathrm{~g}$ for $10 \mathrm{~min}$ at room temperature and the serum was stored at $-80^{\circ} \mathrm{C}$ until analysis.

\section{Analytical methods}

The ex vivo oxidation of isolated LDL was performed by using a modified method of Esterbauer et al. (1989) which has been described previously (Bub et al. 2000). Intra- and inter-assay variability for LDL oxidation was $<5 \%$ and $<8 \%$, respectively. Plasma malondialdehyde (MDA) was measured as thiobarbituric acid reactive substances (TBARS) by HPLC with fluorescence detection (Burkart et al. 2000) with minor modifications as described previously (Briviba et al. 2004). Intra- and inter-assay variabilities were $<5 \%$. Serum triacylglycerol, cholesterol and HDL-cholesterol were determined by enzymatic kits (Boehringer, Mannheim, Germany). LDL-cholesterol was calculated using the 'Friedewald' formula.

\section{PON activity}

Arylesterase activity of PON was determined spectrophotometrically using phenylacetate as substrate as described by Gan et al. (1991) with minor modifications. The assay mixture contained $5.0 \mathrm{mmol} / \mathrm{l}$ of phenylacetate and $0.9 \mathrm{mmol} / 1 \mathrm{CaCl}_{2}$ in $20 \mathrm{mmol} / \mathrm{l}$ Tris- $\mathrm{HCl}, \mathrm{pH} 8.0$, at $25^{\circ} \mathrm{C}$. The reaction was recorded at $270 \mathrm{~nm}$. Non-enzymatic hydrolysis of phenylacetate was subtracted from the total rate of hydrolysis. Results are expressed as $\mathrm{U} / \mathrm{ml}$. The $E_{270}$ for the reaction is $1310 \mathrm{~mol} / \mathrm{l}$ per $\mathrm{cm}$ and $1 \mathrm{U}$ of arylesterase activity is equal to $1 \mu \mathrm{mol}$ of phenylacetate hydrolysed/min per $\mathrm{ml}$. The activity of PON towards paraoxon (paraoxonase activity) was measured as described by Mackness et al. (1998b). Serum was added to Tris- $\mathrm{HCl}$ buffer $(100 \mathrm{mmol} / \mathrm{l}, \mathrm{pH}$ $8.0)$ containing $2 \mathrm{mmol} / \mathrm{l} \mathrm{CaCl}_{2}$ and $5.5 \mathrm{mmol} / \mathrm{l}$ paraoxon (diethyl-p-nitrophenylphosphate; Sigma-Aldrich, Steinheim, Germany). The rate of $p$-nitrophenol generation during incubation at $25^{\circ} \mathrm{C}$ was monitored at $405 \mathrm{~nm}$. One $U$ of paraoxonase activity is equal to $1 \mathrm{nmol}$ of $p$-nitrophenol formed/min per ml. Intra- and inter-assay variabilities were $<5 \%$.

\section{Determination of the paraoxonase 192 genotype}

The PON1-192 genotype was determined by restriction fragment length polymorphism-polymerase chain reaction (RFLP-PCR) using a previously published protocol (Humbert et al. 1993) with some minor modifications as described earlier (Bub et al. 2002). Each genotype was read by two independent observers.

\section{Statistical analysis}

Since genotyping revealed only two subjects to be homozygous for the R-allele, we merged the $\mathrm{QR}$ and the RR subjects to a new group $\mathrm{QR} / \mathrm{RR}$ for further analysis. The cross-over trial was analysed using a standard method for the AB/BA design (Altman, 1991). As sequence effects were not significant, data from both intervention groups (group A: tomato/carrot juice, group B: carrot/tomato juice) were merged and analysed for PON genotype and vegetable juice consumption, respectively. Possible differences at baseline among treatment groups or among PON genotype groups were compared by unpaired $t$ tests. Treatment effects were calculated as the difference between post- and pre-experimental values; within group treatment effects for tomato and carrot juice were tested by one-sample $t$ test; between group treatment effects for tomato $v$. carrot juice were tested by unpaired $t$ test. Comparison of PON genotypes within 
the tomato or carrot juice group were performed using ANOVA with 'vegetable juice' and 'genotype' as factor and the TukeyKramer post hoc test. All statistical calculations were performed with the StatView program (SAS Institute, Cary, NC, USA); significance level was set at $P \leq 0.05$. Results are given as means with their standard deviations.

\section{Results}

All subjects complied with the study protocol and completed both dietary treatments. None of the volunteers reported any illness during the study. PON1-192 genotyping revealed seven subjects carrying the QQ-genotype, thirteen the QR-genotype and two the RR-genotype. The $\mathrm{QR}$ and the RR group have been merged and results are given for the QR/RR group (Table 1). At baseline, paraoxonase activity of PON was 123 (SD 29) and 217 (SD 44) U/l in QQ and $\mathrm{QR} / \mathrm{RR}$ subjects, respectively $(P<0 \cdot 001)$. Arylesterase activity was not significantly different in QQ and $Q R / R R$ subjects (156 (SD 26) and 144 (SD 19) U/ml). Tomato and carrot juice consumption had no statistically significant effects on arylesterase and paraoxonase activity of PON (Table 2). Additionally, tomato and carrot juice consumption had no effect on PON activity in either $\mathrm{QQ}$ or $\mathrm{QR} / \mathrm{RR}$ subjects. We also observed no effects on total cholesterol, LDL-cholesterol or HDL-cholesterol in serum (data not shown).

Previous results from this study showed that tomato and carrot juice consumption had only marginal effects on lipid peroxidation in plasma (Briviba et al. 2004). Since PON can prevent lipid peroxidation depending on the PON genotype, we reanalysed our lipid peroxidation data with focus on the PON genotype. At baseline, lag time was significantly higher during LDL oxidation in QQ than in QR/RR subjects (111 (SD 9) v. 101 (SD 8) min; $P<0 \cdot 05$ ), while MDA in plasma was not significantly different

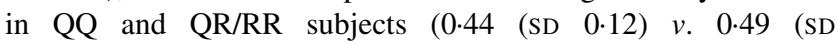
$0 \cdot 18) \mu M$ ). Tomato and carrot juice consumption had no significant effect on lag time in QQ or QR/RR subjects (Fig. 1(A)). However, tomato juice consumption significantly reduced plasma MDA in $\mathrm{QR} / \mathrm{RR}$ subjects $(P<0.05$; Fig. 1(B)), while carrot juice had no significant effect on MDA irrespective of the PON1-192 genotype.

In plasma we also analysed lycopene and $\beta$-carotene, the major carotenoids in tomato juice and carrot juice, respectively. Results are given in Table 3 . Tomato juice consumption significantly increased plasma lycopene concentrations $(P<0 \cdot 001)$, while $\beta$-carotene in plasma did not change significantly. Carrot juice consumption increased $\beta$-carotene in plasma significantly $(P<0.001)$, but had no effect on lycopene plasma concentrations. Before carrot juice consumption plasma lycopene concentrations were lower in $Q R / R R$ subjects compared with QQ subjects $(P=0 \cdot 04)$. However, after carrot juice consumption plasma lycopene significantly decreased in QQ subjects but not in the QR/RR group $(P=0.016)$. Although $\beta$-carotene plasma concentrations decreased during tomato juice consumption, these effects were not significant.

We also compared the data of washout phases at the beginning, during and after intervention (Table 4). There were no significant differences among the washout phases for lag time, plasma MDA, PON activities and $\beta$-carotene plasma concentrations. However, plasma lycopene concentrations were significantly lower $(P<0.05)$ at the end of the last washout phase.

\section{Discussion}

Supplementation of a low-carotenoid diet with either tomato or carrot juice for 2 weeks had no effect on the arylesterase and paraoxonase activity of PON in healthy young men. This is in line with a previous report which showed that tomato juice consumption over a period of 8 weeks had no effect on arylesterase activity in healthy elderly people (Bub et al. 2002). Other groups, however, have found that arylesterase activity increased after consumption of pomegranate juice (Aviram et al. 2000) and alcoholic beverages (van der Gaag et al. 1999) but decreased after used cooking fat (Sutherland et al. 1999).

The PON1-192 genotype predicts paraoxonase activity in human serum (Humbert et al. 1993). Our data on differences in basal paraoxonase activity are in line with this observation: low paraoxonase activity in QQ subjects, high activity in RR subjects and intermediate activity in QR heterozygeous volunteers. We found no effect of tomato or carrot juice consumption on paraoxonase activity of PON1 in men regardless of PON1-192 genotype. Recently, it has been shown that a 5-week diet high in vegetables reduced paraoxonase activity in women, especially in those with the QR and RR genotype (Rantala et al. 2002). The authors discussed the possibility that changes in dietary fatty acid intake and changes in HDL-cholesterol may partly account for the observed effects. In our study HDL-cholesterol was unaffected, which may explain why PON activity was unchanged. Sex differences may also contribute to the observed discrepancies between our study

Table 1. Baseline characteristics of study participants based on treatment groups and on PON1 polymorphism†

\begin{tabular}{|c|c|c|c|c|c|c|c|c|c|c|}
\hline & & & \multicolumn{4}{|c|}{ Intervention groups } & \multicolumn{4}{|c|}{ PON1 polymorphism } \\
\hline & \multicolumn{2}{|c|}{ Total (n 22) } & \multicolumn{2}{|c|}{ Group Ał (n 11) } & \multicolumn{2}{|c|}{ Group B (n 11) } & \multicolumn{2}{|c|}{ QQ§ $(n 7)$} & \multicolumn{2}{|c|}{ QR/RR ( $n$ 15) } \\
\hline & Mean & $\mathrm{SD}$ & Mean & SD & Mean & SD & Mean & SD & Mean & SD \\
\hline Age (years) & 29 & 6 & 30 & 7 & 28 & 5 & 29 & 8 & 29 & 5 \\
\hline BMI $\left(\mathrm{kg} / \mathrm{m}^{2}\right)$ & 23 & 2 & 22 & 2 & 24 & 2 & 23 & 2 & 23 & 2 \\
\hline Cholesterol (mmol/l) & 4.4 & 0.9 & $4 \cdot 1$ & 0.8 & 4.6 & 1.0 & 4.2 & 0.9 & 4.4 & 1.0 \\
\hline LDL-cholesterol (mmol/l) & $2 \cdot 5$ & 0.7 & $2 \cdot 3$ & 0.7 & $2 \cdot 6$ & 0.7 & $2 \cdot 4$ & 0.8 & $2 \cdot 5$ & 0.6 \\
\hline HDL-cholesterol (mmol/l) & $1 \cdot 3$ & 0.3 & 1.2 & 0.3 & $1 \cdot 3$ & 0.3 & $1 \cdot 3$ & 0.3 & $1 \cdot 2$ & 0.3 \\
\hline
\end{tabular}

†There were no significant differences at baseline among groups $A$ and $B$ or $Q Q$ and QR/RR, respectively (unpaired $t$ test).

$\ddagger$ Group $A$ denotes the subgroup of subjects after randomization with the intervention sequence tomato juice/carrot juice; group B consumed carrot juice followed by tomato juice. $\S Q Q$ indicates homozygous subjects with glutamine at position 192 of the PON1 gene; QR/RR are heterozygous and homozygous, respectively, with arginine substituted for glutamine. 
Table 2. Effect of tomato and carrot juice consumption for 2 weeks on arylesterase and paraoxonase activities of human serum paraoxonase in healthy men with respect to PON1 genotype†

(Mean values and standard deviations)

\begin{tabular}{|c|c|c|c|c|c|c|c|c|c|c|c|c|}
\hline & \multicolumn{6}{|c|}{ Before intervention } & \multicolumn{6}{|c|}{ After intervention } \\
\hline & \multicolumn{2}{|c|}{ Total (n 22) } & \multicolumn{2}{|c|}{$\mathrm{QQ \S}(n 7)$} & \multicolumn{2}{|c|}{ QR/RR ( $n$ 15) } & \multicolumn{2}{|c|}{ Total $(n 22)$} & \multicolumn{2}{|c|}{$\mathrm{QQ}(n 7)$} & \multicolumn{2}{|c|}{ QR/RR ( $n$ 15) } \\
\hline & Mean & SD & Mean & SD & Mean & SD & Mean & SD & Mean & SD & Mean & SD \\
\hline \multicolumn{13}{|l|}{ Tomato juice } \\
\hline Arylesterase $(\mathrm{U} / \mathrm{ml}) \ddagger$ & 147 & 25 & 147 & 34 & 142 & 19 & 146 & 24 & 161 & 26 & 140 & 20 \\
\hline Paraoxonase $(\mathrm{U} / \mathrm{I})$ & 185 & 60 & 123 & 29 & $213^{*}$ & 48 & 181 & 54 & 126 & 27 & $207^{\star}$ & 43 \\
\hline Arylesterase (U/ml) & 146 & 23 & 154 & 28 & 142 & 19 & 148 & 20 & 154 & 22 & 145 & 20 \\
\hline Paraoxonase (U/I) & 190 & 62 & 124 & 28 & $220^{*}$ & 47 & 187 & 62 & 118 & 18 & $220^{*}$ & 46 \\
\hline
\end{tabular}

${ }^{*} P<0.001$. Subjects carrying the R-allele ( $Q R$ and RR) have a significantly higher paraoxonase activity as compared to the QQ wild-type (unpaired $t$ test) before and after intervention, respectively.

†There were no significant differences at baseline among the tomato juice group and the carrot juice group for arylesterase and paraoxonase activity, respectively (unpaired $t$ test). Intervention with either juice had no significant effect on enzyme activities. Intervention effects were calculated by subtracting pre- from post-experimental values and tested by one-sample $t$ test within each juice intervention group. Unpaired $t$ test was applied to compare treatment effects induced by tomato juice and carrot juice.

$\ddagger$ PON1 activity has been determined by using phenylacetate (arylesterase) and paraoxon (paraoxonase) as substrates, respectively. One $U$ of arylesterase activity is equal to $1 \mu$ mol phenylacetate hydrolysed/min per $\mathrm{ml}$. One $U$ of paraoxonase activity is equal to $1 \mathrm{nmol}$ paraoxon hydrolysed/min per $\mathrm{ml}$.

$\S \mathrm{Q}$ indicates homozygous subjects with glutamine at position 192 of the PON1 gene; QR/RR are heterozygous and homozygous, respectively, with arginine substituted for glutamine.
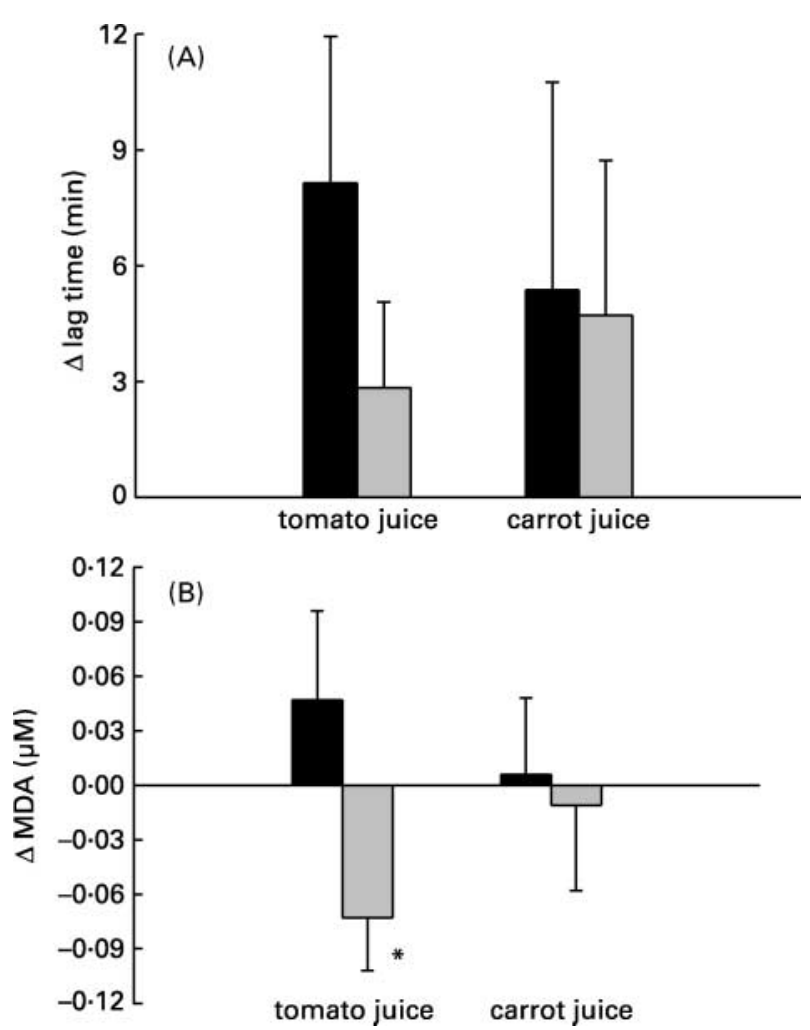

Fig. 1. Changes $(\Delta)$ in lag time $(A)$ as a measure of LDL oxidation, and plasma malondialdehyde (MDA) (B) in men after drinking $330 \mathrm{ml}$ tomato or carrot juice for 2 weeks, respectively. Lag time reflects the antioxidant status of LDL and is determined by monitoring conjugated diene formation during copper-induced LDL oxidation. It is defined as the time from reaction start to the beginning of the rapid increase in diene production (propagation phase). Values are means with their standard deviations represented by vertical

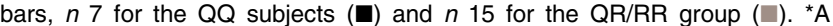
significant reduction in plasma MDA was detected after tomato juice consumption (ANOVA, Tukey-Kramer test, $P<0.05$ ). QQ indicates homozygous subjects with glutamine at position 192 of the PON gene. QR/RR are heterozygous and homozygous, respectively, with arginine substituted for glutamine. with men and the study by Rantala with women. Paraoxonase activity is higher in women than in men on a habitual diet (Kleemola et al. 2002). The authors found a significant negative correlation between paraoxonase activity and vegetable, fruit and berry intake in women only and they discussed the hormonal status which they thought might be responsible for the observed sex differences. Unfortunately, they did not determine the PON1192 genotype which is a predictor of paraoxonase activity (Humbert et al. 1993), and could also account for the observed genderrelated differences.

Consumption of tomato products reduces lipid peroxidation in healthy volunteers (Agarwal \& Rao, 1998; Bub et al. 2000; Visioli et al. 2003). However, in the present study tomato and carrot juice consumption had only marginal effects on lipid peroxidation in plasma (Briviba et al. 2004). We therefore reanalysed our data focusing on the respective PON genotypes. At baseline we found that lag time was shorter in $\mathrm{QR} / \mathrm{RR}$ subjects than in QQ subjects, thus LDL oxidation in the QR/RR group was higher. HDL-associated PON1 protects LDL from oxidation as seen by reduced lipid peroxide formation (Mackness et al. 1998b). Although R-allele carriers show higher paraoxonase activity of PON1, lipid peroxide degradation is lower than in the QQ genotype (Mackness et al. $2000 b$ ), which might explain why lag time was shorter in the QR/RR subjects. MDA, an end product of lipid peroxidation, circulates in plasma. One would expect an inverse association between lag time and plasma MDA. However, despite lower lag time in the QR/RR group, plasma MDA was not significantly higher in this group before intervention (QR/RR: 0.49 (SD 0.18); QQ:0.44 (SD 0.12); $P=0.51)$. It is therefore doubtful whether, under the given study conditions, there is any link between plasma MDA and LDL oxidation in healthy volunteers.

The differences in lag time before intervention may be partly attributable to differences in plasma carotenoid concentrations. Carotenoids are potent antioxidants and can inhibit lipid peroxidation (Krinsky 1998). High concentrations of carotenoids in plasma at baseline could protect LDL against oxidation. Lycopene and $\beta$-carotene in plasma tended to be lower in the $\mathrm{QR} /$ $\mathrm{RR}$ group than in $\mathrm{QQ}$ subjects, even before intervention, and this may possibly contribute to the lower lag time in the QR/ 
Table 3. Plasma carotenoid concentrations in healthy men with respect to PON1 genotype after tomato and carrot juice consumption for 2 weeks

(Mean values and standard deviations)

\begin{tabular}{|c|c|c|c|c|c|c|c|c|c|c|c|c|c|c|}
\hline & \multicolumn{7}{|c|}{ Before intervention } & \multicolumn{7}{|c|}{ After intervention } \\
\hline & \multicolumn{2}{|c|}{ Total $(n 22)$} & \multicolumn{2}{|c|}{ QQ† $(n 7)$} & \multicolumn{2}{|c|}{$\mathrm{QR} / \mathrm{RR}(n 15)$} & \multirow[b]{2}{*}{$P \ddagger$} & \multicolumn{2}{|c|}{ Total (n 22) } & \multicolumn{2}{|c|}{$\mathrm{QQ}(n 7)$} & \multicolumn{2}{|c|}{ QR/RR ( $n$ 15) } & \multirow[b]{2}{*}{$P \S$} \\
\hline & Mean & SD & Mean & SD & Mean & SD & & Mean & SD & Mean & SD & Mean & SD & \\
\hline \multicolumn{15}{|l|}{ Tomato juice } \\
\hline Lycopene (nmol/l) & 195 & 184 & 228 & 169 & 180 & 194 & NS & $464^{*}$ & 226 & 559 & 251 & 420 & 208 & NS \\
\hline$\beta$-Carotene (nmol/l) & 366 & 212 & 423 & 229 & 340 & 207 & NS & 308 & 187 & 367 & 193 & 280 & 184 & NS \\
\hline Lycopene (nmol/l) & 215 & 96 & 275 & 86 & 187 & 89 & 0.04 & 153 & 103 & 139 & 71 & 167 & 117 & 0.016 \\
\hline$\beta$-Carotene $(\mathrm{nmol} / \mathrm{l})$ & 234 & 181 & 271 & 144 & 216 & 198 & NS & $1120^{*}$ & 450 & 1297 & 466 & 1038 & 433 & NS \\
\hline
\end{tabular}

${ }^{*} P<0.001$ for plasma carotenoid concentrations after juice consumption compaired with baseline data.

†QQ indicates homozygous subjects with glutamine at position 192 of the PON1 gene; QR/RR are heterozygous and homozygous, respectively, with arginine substituted for glutamine. $\ddagger$ Differences among the QQ group and the QR/RR group for plasma lycopene and $\beta$-carotene concentrations, respectively, before juice consumption (unpaired $t$ test).

$\S$ Comparison of the QQ group and the QR/RR group for intervention effects is given. Intervention effects were calculated by subtracting pre- from post-experimental values. Unpaired $t$ test was applied to compare intervention effects between the $Q Q$ group and the QR/RR group.

Table 4. Comparison of the washout phases for lag time, malondialdehyde, PON1 activities, and carotenoid concentrations in healthy men on a low carotenoid diet†

(Mean values and standard deviations for 22 subjects)

\begin{tabular}{|c|c|c|c|c|c|c|}
\hline & \multicolumn{2}{|c|}{ Washout $1 \mp$} & \multicolumn{2}{|c|}{ Washout 2} & \multicolumn{2}{|c|}{ Washout 3} \\
\hline & Mean & SD & Mean & SD & Mean & SD \\
\hline Lag time $(\min ) \S$ & 104 & 10 & 105 & 10 & 103 & 13 \\
\hline Malondialdehye $(\mu \mathrm{M})$ & 0.47 & 0.16 & 0.42 & 0.17 & 0.44 & 0.14 \\
\hline Arylesterase (U/ml) & 148 & 22 & 145 & 25 & 142 & 20 \\
\hline Paraoxonase (U/I) & 187 & 60 & 187 & 63 & ND & \\
\hline Lycopene $(\mu \mathrm{M})$ & 0.22 & 0.11 & 0.17 & $0 \cdot 10$ & $0.14^{*}$ & 0.08 \\
\hline$\beta$-Carotene $(\mu \mathrm{M})$ & 0.26 & 0.20 & 0.34 & 0.21 & 0.32 & 0.23 \\
\hline
\end{tabular}

ND, not determined due to technical reasons.

* Lycopene plasma concentrations were significantly lower at the end of washout 3 compared with washout 1 (ANOVA, $P<0.05$ ).

†PON1 activity has been determined by using phenylacetate (arylesterase) and paraoxon (paraoxonase) as substrates, respectively. One $U$ of arylesterase activity is equal to $1 \mu \mathrm{mol}$ phenylacetate hydrolysed/min per $\mathrm{ml}$. One $\mathrm{U}$ of paraoxonase activity is equal to $1 \mathrm{nmol}$ paraoxon hydrolysed/min per $\mathrm{ml}$.

$\ddagger$ Volunteers were on a low carotenoid diet throughout the study. Washout 1 was the run-in period before the first intervention period. Washout 2 was between intervention periods 1 and 2 . Washout 3 followed the second intervention period.

$\S$ Lag time reflects the antioxidant status of LDL and is determined by monitoring conjugated diene formation during copper-induced LDL oxidation. It is defined as the time from reaction start to the beginning of the rapid increase in diene production.

RR group. However, we cannot rule out the possibility that other factors, e.g. vitamin E, may be responsible for the lower lag time in R-allele carriers. Vitamin E plays a crucial role in the protection of LDL from oxidation and the pathogenesis and progression of atherosclerosis (Salonen, 2002; Upston et al. 2003). Unfortunately, we did not determine vitamin E plasma concentrations in our volunteers. However, in a previous study supplementation with identical juice preparations had no significant effect on plasma vitamin $E$ of healthy men (Müller et al. 1999). In a study with elderly men and women we found no significant differences in lag time or plasma MDA between the two genotypes before intervention (Bub et al. 2002), suggesting that age and gender possibly contribute to the observed genotype-related differences.

So far little is known about the impact of dietary intervention on lipid peroxidation in healthy volunteers when PON polymorphisms are considered. In a study with elderly subjects (Bub et al. 2002) we found that tomato juice consumption increased lag time and reduced plasma MDA in QR/RR subjects. This is in line with the results of the present study in healthy young volunteers. Tomato juice consumption reduced plasma MDA in the $\mathrm{QR} / \mathrm{RR}$ group more than in the QQ volunteers. However, there were no significant differences in lag time between the two groups. The relatively short intervention period ( 2 weeks rather than 2 months in the study with elderly subjects) and the small number of volunteers (twenty-two rather than twenty-nine) may explain the absence of an effect on lag time in this study. Carrot juice consumption had no effect on lag time or on plasma MDA in either $\mathrm{QQ}$ or $\mathrm{QR} / \mathrm{RR}$ subjects, despite the fact that it led to plasma $\beta$-carotene concentrations which were twice as high as plasma lycopene concentrations following tomato juice consumption. Carrot juice intake and subsequent high $\beta$-carotene plasma concentrations per se may not be sufficient to protect LDL from oxidation in healthy men. Additionally, the copper-induced ex vivo oxidation of LDL used in this study may not be an adequate measure of LDL oxidizability since it does not reflect physiological in vivo conditions and may mask possible protective effects attributable to carrot juice consumption.

During the entire study the volunteers were kept on a low-carotenoid diet which has been shown to lower plasma carotenoid concentrations after 10 weeks (Watzl et al. 2003). Depletion of carotenoids (and probably other nutrients from fruit and vegetables) may induce 'stress' which might not be completely 
alleviated by supplementation with carrot and tomato juice. This could explain the absence of effects on lag time and MDA after carrot juice consumption. However, despite a 10-week-long low-carotenoid diet we found no increase in lipid peroxidation measurements and no negative effect on immune functions.

In order to minimize seasonal effects, we used a crossover study design with washout phases of 2-week duration, before, between and after the intervention periods. Comparing the three washout phases we found no differences in lag phase, MDA or PON1 activities. This shows that our study design was effective in achieving similar conditions for each intervention and that the low-carotenoid diet had no effect on these measurements. However, lycopene plasma concentrations were reduced after the last washout phase, probably due to the low-carotenoid diet and the fact that there are few sources of lycopene in food. Plasma $\beta$-carotene concentrations did not significantly decrease during the study. The widespread occurrence of $\beta$-carotene in food means that even on a low-carotenoid diet a constant intake of small amounts of $\beta$-carotene is probably unavoidable, which could explain why the decrease in $\beta$-carotene after the last washout phase was non-significant.

In conclusion, male volunteers with the $\mathrm{QR} / \mathrm{RR}$ genotype showed increased lipid peroxidation under baseline conditions. Although tomato and carrot juice failed to affect PON1 activity, tomato juice did reduce lipid peroxidation in volunteers with the PON1-192 R-allele, and could therefore play a useful role in reducing the risk of CVD in this subgroup. The reduction in lipid peroxidation after tomato juice consumption appeared to be more pronounced in RR homozygous subjects but as we had only two volunteers with this genotype further studies with an increased study sample would be needed to ascertain the significance of this finding.

\section{Acknowledgements}

This study was supported by a grant from the 'Deutsche Krebshilfe' (project \#70-2165). The authors thank M. Broßart, T. Gadau, D. Haase and U. Stadler-Prayle for their excellent technical assistance and the volunteers for taking part in this study. Tomato juice was kindly provided by Schoenenberger Pflanzensäfte GmbH, Magstadt, Germany.

\section{References}

Adkins S, Gan KN, Mody M \& La Du BN (1993) Molecular basis for the polymorphic forms of human serum paraoxonase/ arylesterase: glutamine or arginine at position 191, for the respective A or B alloenzyme. Am J Hum Genet 52, 598-608.

Agarwal S \& Rao AV (1998) Tomato lycopene and low density lipoprotein oxidation: a human dietary intervention study. Lipids 33, 981-984.

Altman DG (1991) Clinical trials. In Practical Statistics for Medical Research, pp. 440-476 [DG Altman, editor]. London: Chapman \& Hall.

Aviram M (1999) Does paraoxonase play a role in susceptibility to cardiovascular disease? Mol Med Today 5, 381-386.

Aviram M, Billecke S, Sorenson R, Bisgaier CL, Newton R, Rosenblat M, Erogul J, Hsu C, Dunlop C \& La Du B (1998) Paraoxonase active site required for protection against LDL oxidation involves its free sulfhydryl group and is different from that required for its arylesterase/paraoxonase activities. Arterioscler Thromb Vasc Biol 18, 1617-1624.

Aviram M, Dornfeld L, Rosenblat M, Volkova N, Kaplan M, Colemann R, Hayek T, Presser D \& Fuhrman B (2000) Pomegranate juice consumption reduces oxidative stress, atherogenic modifications to LDL, and platelet aggregation: studies in humans and in atherosclerotic apolipoprotein E-deficient mice. Am J Clin Nutr 71, 1062-1076.

Bazzano LA, He J, Ogden LG, Loria CM, Vupputuri S, Myers L \& Whelton PK (2002) Fruit and vegetable intake and risk of cardiovascular disease in US adults: the first National Health and Nutrition Examination Survey Epidemiologic Follow-up Study. Am J Clin Nutr 76, 93-99.

Briviba K, Schnäbele K, Rechkemmer G \& Bub A (2004) Supplementation of a diet low in carotenoids with tomato and carrot juice does not affect lipid peroxidation in plasma and feces of healthy men. J Nutr 134, 1081-1083.

Bub A, Barth S, Watzl B, Briviba K, Herbert BM, Luhrmann PM, Neuhauser-Berthold M \& Rechkemmer G (2002) Paraoxonase 1 Q192R (PON1-192) polymorphism is associated with reduced lipid peroxidation in R-allele-carrier but not in QQ homozygous elderly subjects on a tomato-rich diet. Eur J Nutr 41, 237-243.

Bub A, Watzl B, Abrahamse L, Delincée H, Adam S, Wever J, Müller H \& Rechkemmer G (2000) Moderate intervention with carotenoid-rich vegetable products reduces lipid peroxidation in men. $J$ Nutr 130, 2200-2206.

Burkart V, Liu H, Bellmann K, Wissing D, Jäättelä M, Cavallo MG, Pozilli P, Briviba K \& Kolb H (2000) Natural resistance of human beta cells toward nitric oxide is mediated by heat shock protein $70 . J$ Biol Chem 275, 19521-19528.

Diaz MN, Frei B, Vita JA \& Keaney JF (1997) Mechanisms of disease: antioxidants and atherosclerotic heart disease. $N$ Engl J Med 337, 408-416.

Esterbauer H, Striegl G, Puhl H \& Rotheneder M (1989) Continuous monitoring of in vitro oxidation of human low density lipoprotein. Free Rad Res Commun 6, 67-75.

Frei B, Keaney JF, Retsky KL \& Chen K (1996) Vitamins C and E and LDL oxidation. In Vitamins and Hormones-Advances in Research and Applications, Vol. 52. pp. 1-34 [G Litwack, editor]. San Diego: Academic Press.

Gan KN, Smolen A, Eckerson HW \& La Du BN (1991) Purification of human serum paraoxonase/arylesterase. Evidence for one esterase catalyzing both activities. Drug Metab Dispos 19, 100-106.

Humbert R, Adler DA, Disteche CM, Hassett C, Omiecinski CJ \& Furlong CE (1993) The molecular basis of the human serum paraoxonase activity polymorphism. Nat Genet 3, 73-76.

Jarvik GP, Hatsukami TS, Carlson C, Richter RJ, Jampsa R, Brophy VH, Margolin S, Rieder M, Nickerson D, Schellenberg GD, Heagerty PJ \& Furlong CE (2003) Paraoxonase activity, but not haplotype utilizing the linkage disequilibrium structure, predicts vascular disease. Arteriosc Thromb Vasc Biol 23, 1465-1471.

Jarvik GP, Rozek LS, Brophy VH, Hatsukami TS, Richter RJ, Schellenberg GD \& Furlong CE (2000) Paraoxonase (PON1) phenotype is a better predictor of vascular disease than is PON1(192) or PON1(55) genotype. Arterioscler Thromb Vasc Biol 20, 2441-2447.

Jialal I \& Devaraj S (1996) Low density lipoprotein oxidation, antioxidants, and atherosclerosis: a clinical biochemistry perspective. Clin Chem 42, 498-506.

Joshipura KJ, Hu FB, Manson JE, et al. (2001) The effect of fruit and vegetable intake on risk for coronary heart disease. Ann Intern Med 134, 1106-1114.

Kleemola P, Freese R, Jauhiainen M, Pahlman R, Alfthan G \& Mutanen M (2002) Dietary determinants of serum paraoxonase activity in healthy humans. Atherosclerosis 160, 425-432.

Krinsky NI (1998) The antioxidant and biological properties of the carotenoids. Ann NY Acad Sci 854, 443-447.

Kudchodkar BJ, Lacko AG, Dory L \& Fungwe TV (2000) Dietary fat modulates serum paraoxonase 1 activity in rats. $J$ Nutr 130, 2427-2433.

Liu S, Lee IM, Ajani U, Cole SR, Buring JE \& Manson JE (2001) Intake of vegetables rich in carotenoids and risk of coronary heart disease in men: The Physicians' Health Study. Int J Epidemiol 30, 130-135. 
Liu S, Manson JE, Lee IM, Cole SR, Hennekens CH, Willett WC \& Buring JE (2000) Fruit and vegetable intake and risk of cardiovascular disease: the Women's Health Study. Am J Clin Nutr 72, 922-928.

Mackness B, Durrington P, McElduff P, Yarnell J, Azam N, Watt M \& Mackness M (2003) Low paraoxonase activity predicts coronary events in the caerphilly prospective study. Circulation 107, 2775-2779.

Mackness B, Mackness MI, Arrol S, Turkie W \& Durrington PN (1998b) Effect of the human serum paraoxonase 55 and 192 genetic polymorphisms on the protection by high density lipoprotein against low density lipoprotein oxidative modification. FEBS Lett 423, 57-60.

Mackness MI, Bouiller A, Hennuyer N, Mackness B, Hall M, Tailleux A, Duriez P, Delfly B, Durrington P, Fruchart JC, Duverger N, Caillaud JM \& Castro G (2000b) Paraoxonase activity is reduced by a pro-atherosclerotic diet in rabbits. Biochem Biophys Res Commun 269, 232-236.

Mackness MI, Durrington PN \& Mackness B (2000a) How high-density lipoprotein protects against the effects of lipid peroxidation. Curr Opin Lipidol 11, 383-388.

Mackness MI, Harty D, Bhatnagar D, Winocour PH, Arrol S, Ishola M \& Durrington PN (1991) Serum paraoxonase activity in familial hypercholesterolaemia and insulin-dependent diabetes mellitus. Atherosclerosis 86, $193-199$.

Mackness MI, Mackness B, Durrington PN, Fogelman AM, Berliner JXLA, Navab M, Shih D \& Fonarow GC (1998a) Paraoxonase and coronary heart disease. Curr Opin Lipidol 9, 319-324.

Müller H, Bub A, Watzl B \& Rechkemmer G (1999) Plasma concentrations of carotenoids in healthy volunteers after intervention with carotenoid-rich foods. Eur J Nutr 38, 35-44.

Osganian SK, Stampfer MJ, Rimm E, Spiegelman D, Manson JE \& Willett WC (2003) Dietary carotenoids and risk of coronary artery disease in women. Am J Clin Nutr 77, 1390-1399.
Rantala M, Silaste ML, Tuominen A, Kaikkonen J, Salonen JT, Alfthan G, Aro A \& Kesäniemi YA (2002) Dietary modifications and gene polymorphisms alter serum paraoxonase activity in healthy women. J Nutr 132, 3012-3017.

Salonen JT (2002) Clinical trials testing cardiovascular benefits of antioxidant supplementation. Free Rad Res 36, 1299-1306.

Sutherland WHF, Walker RJ, DeJong SA, vanRijn AM, Phillips V \& Walker HL (1999) Reduced postprandial serum paraoxonase activity after a meal rich in used cooking fat. Arterioscler Thromb Vasc Biol 19, $1340-1347$.

Tomás M, Sentí M, Elosua R, Vila J, Sala J, Masià R \& Marrugat J (2001) Interaction between the Gln-Arg 192 variants of the paraoxonase gene and oleic acid intake as a determinant of high-density lipoprotein cholesterol and paraoxonase activity. Eur J Pharmacol 432, 121-128.

Upston JM, Kritharides L \& Stocker R (2003) The role of vitamin E in atherosclerosis. Prog Lip Res 42, 405-422.

van der Gaag MS, van Tol A, Scheek LM, James RW, Urgert R, Schaafsma G \& Hendriks HFJ (1999) Daily moderate alcohol consumption increases serum paraoxonase activity; a diet-controlled, randomized intervention study in middle-aged men. Atherosclerosis 147, 405-410.

Visioli F, Riso P, Grande S, Galli C \& Porrini M (2003) Protective activity of tomato products on in vivo markers of lipid oxidation. Eur J Nutr $\mathbf{4 2}$, 201-206.

Vivekananthan DP, Penn MS, Sapp SK, Hsu A \& Topol EJ (2003) Use of antioxidant vitamins for the prevention of cardiovascular disease: metaanalysis of randomised trials. Lancet 361, 2017-2023.

Watzl B, Bub A, Briviba K \& Rechkemmer G (2003) Supplementation of a low-carotenoid diet with tomato or carrot juice modulates immune functions in healthy men. Ann Nutr Metab 47, 255-261. 